\title{
On the formation of neon-enriched donor stars in ultracompact $\mathrm{X}$-ray binaries
}

\author{
L. R. Yungelson ${ }^{1,2}$, G. Nelemans ${ }^{3}$, and E. P. J. van den Heuvel ${ }^{2}$ \\ 1 Institute of Astronomy of the Russian Academy of Sciences, 48 Pyatnitskaya Str., 119017 Moscow, Russia \\ 2 Astronomical Institute "Anton Pannekoek", Kruislaan 403, 1098 SJ Amsterdam, The Netherlands \\ 3 Institute of Astronomy, University of Cambridge, Madingley Road, CB3 0HA, Cambridge, UK
}

Received 21 December 2001 / Accepted 3 April 2002

\begin{abstract}
We study the formation of neon-enriched donor stars in ultracompact X-ray binaries $\left(P_{\text {orb }}<80\right.$ min $)$ and show that their progenitors have to be low-mass $(0.3-0.4) M_{\odot}$ "hybrid" white dwarfs (with CO cores and thick helium mantles). Stable mass transfer is possible if in the initial stages of mass exchange mass is lost from the system, taking away the specific orbital angular momentum of the accretor ("isotropic re-emission"). The excess of neon in the transferred matter is due to chemical fractionation of the white dwarf which has to occur prior to the Roche lobe overflow by the donor. The estimated lower limit of the orbital periods of the systems with neon-enriched donors is close to $10 \mathrm{~min}$. We show that the X-ray pulsar 4U 1626-67, which likely also has a neon-enriched companion, may have been formed via accretion induced collapse of an oxygen-neon white dwarf accretor if the donor was a hybrid white dwarf.
\end{abstract}

Key words. stars: white dwarfs - stars: mass loss - stars: abundances - binaries: close - X-rays: binaries

\section{Introduction}

Recently, Juett et al. (2001) reported the discovery of the excess of neon in four low-mass X-ray binaries: $4 \mathrm{U} 0614+091,2 \mathrm{~S}$ 0918-549, $4 \mathrm{U}$ 1543-624, and $4 \mathrm{U}$ 1850-0857. It is suggested that the Ne is local to these objects. $4 \mathrm{U}$ 1850-0857, which belongs to a globular cluster, has a measured orbital period of 20.6 min. From the optical properties of the systems, Juett et al. suggest that all of them are ultracompact: $P_{\text {orb }}<80 \mathrm{~min}$. A local excess of neon is found also in the ultracompact $\left(P_{\text {orb }}=41.4 \mathrm{~min}\right)$ binary pulsar $4 \mathrm{U}$ 1626-67 (Angelini et al. 1995; Schulz et al. 2001). Juett et al. propose that in all these binaries the donors are low-mass, neon-rich degenerate dwarfs. Schulz et al. (2001) suggest that the donor in $4 \mathrm{U}$ 1626-67 is the core of a carbon-oxygen (CO) or oxygen-neon $(\mathrm{ONe})$ white dwarf which has previously crystallised. However, the evolution of neutron star+white dwarf [henceforth, (wd, ns)] binaries to the Ne-enrichment stage was not studied.

Below, we explore the evolution of white dwarfs which fill their Roche lobes having neutron star companions. We discuss the mechanism of mass exchange and

Send offprint requests to: L. Yungelson,

e-mail: lry@inasan.rssi.ru limiting masses of the donors in these binaries in Sect. 2 . The process of chemical fractionation that could lead to Ne-enrichment is briefly outlined in Sect. 2.3. The observed systems are discussed in Sect. 3. Some uncertainties of the model and related problems are considered in Sect. 4. Our conclusions follow in Sect. 5 .

\section{Evolution of ultracompact binaries}

\subsection{Formation of ultracompact binaries}

After pioneering work of Paczyński (1967) on the ultrashort-period ( $18 \mathrm{~min})$ cataclysmic variable AM CVn, it is commonly accepted that the donors in semi-detached systems with the orbital periods of several tens of minutes may be degenerate dwarfs and that the driving force of the binary evolution is angular momentum loss (AML) due to gravitational wave radiation (GWR).

The basic features of the scenario for the formation of (wd, ns) systems which can evolve into ultracompact X-ray binaries may be summarized as follows (see e.g. Tutukov \& Yungelson 1993; Iben et al. 1995, for details). In the Galactic disk stars with masses $>10 M_{\odot}$ that are born in binaries evolve into neutron stars, experiencing underway one or two common envelope stages which strongly decrease the orbital separation. Next, their companions 
CO donor+neutron star accretor

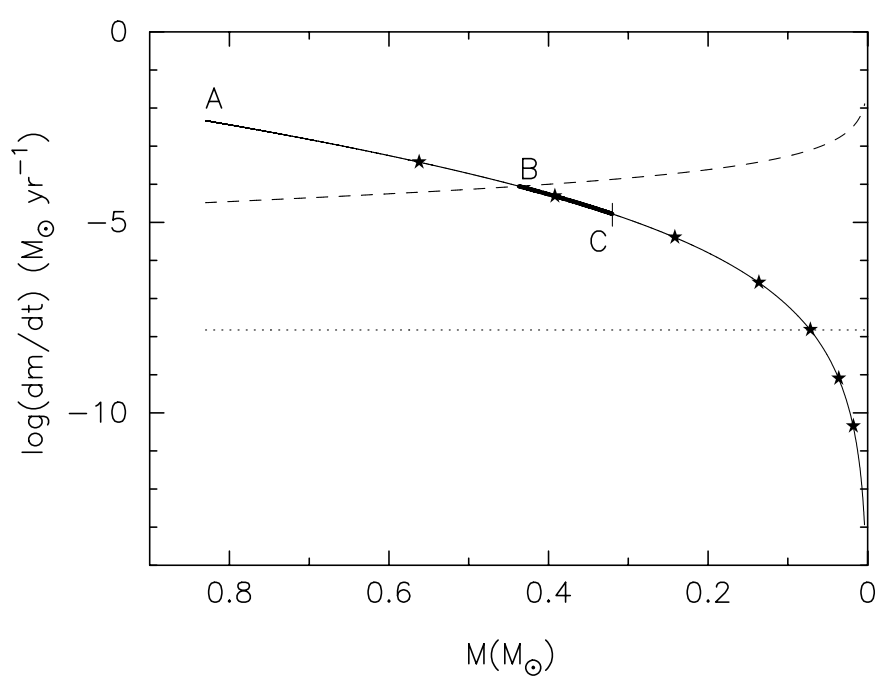

Fig. 1. The solid line shows the mass loss rate from a white dwarf "stabilised" by isotropic re-emission for the case of a system which initially contained a $0.83 M_{\odot}$ white dwarf donor and a $1.433 M_{\odot}$ neutron star accretor. Dashed line: the upper limit of the mass loss rate from the system by the isotropic re-emission (for $r_{\mathrm{ns}}=10 \mathrm{~km}$ ). Dotted line: $\dot{M}_{\text {Edd }}$ for a neutron star. Asterisks mark the parameters of the system at $\log t(\mathrm{yr})=3,4,5,6,7,8,9$. Systems with different initial $M_{\mathrm{d}}$ follow curves which are indistinguishable on the scale of the figure. To the left of the beginning of the mass-loss curve (A) the Roche-lobe filling white dwarfs are dynamically unstable. Between A and B isotropic re-emission is not efficient enough for ejection of all matter lost by white dwarf. "Hybrid" white dwarfs which are able to transfer matter stably have initial masses corresponding to the thick part of the mass-loss line BC.

experience dynamically unstable mass loss and become white dwarfs. If the resulting systems have orbital separation $\sim R_{\odot}$, AML via GWR may bring the white dwarf to Roche-lobe overflow (RLOF) within the lifetime of the Galactic disk and under certain conditions (see below) stable mass exchange leading to exposure of the Ne-enriched core may ensue.

In globular clusters neutron stars most probably acquire their companions via exchange interactions with primordial binaries (e.g. Rasio et al. 2000). The subsequent evolution is similar to that of the disk binaries.

\subsection{Mass transfer in ultracompact binaries: The nature of the donors and the limits of their initial mass}

In systems with degenerate donors, at the onset of Rochelobe overflow the mass transfer rate $\dot{M}$ may be several orders of magnitude higher than the Eddington limiting accretion rate $\left(\dot{M}_{\text {Edd }}\right)$ for a white dwarf or neutron star accretor (Vila 1971; Tutukov \& Yungelson 1979), (see Fig. 1). Formation of a common envelope engulfing the whole system and merger of components or collapse of the neutron star into a black hole may be avoided if the excess of the matter which cannot be accreted leaves the system taking away the specific orbital angular momentum of the accreting component ("isotropic re-emission" Soberman et al. 1997). This role may be accomplished by radiatively driven outflows (King \& Begelman 1999; Tauris \& Savonije 1999). The equation for the mass loss rate by a white dwarf in the presence of AML by GWR and "isotropic re-emission" used in this study may be found in Yungelson et al. (1996).

Figure 1 shows this mass loss rate for a (wd, ns) system with initial masses of donor and accretor $M_{\mathrm{d}}=0.83 M_{\odot}$ and $M_{\mathrm{a}}=1.433 M_{\odot}$, respectively ${ }^{1}$. Since all white dwarfs approach zero-temperature radii after cooling for several 100 Myr (Panei et al. 2000) and since most systems will be very much older than this when their orbits have decayed sufficiently to start mass transfer, we use in our computations the mass - radius $(M-R)$ relation for cold spheres derived by Zapolsky \& Salpeter (1969) in the form given by Rappaport et al. (1987), assuming equal mass fractions of carbon and oxygen. For this $M-R$ relation, if the mass retention efficiency of the accretor is close to zero, (wd, ns) systems with $M_{\mathrm{d}} \gtrsim 0.83 M_{\odot}$ are dynamically unstable.

All matter in excess of $\dot{M}_{\text {Edd }}$ can be lost from the system only if the liberated accretion energy of the matter falling from the Roche lobe radius of the neutron star to the neutron star surface is sufficient to expel the matter from the Roche-lobe surface around the neutron star, i.e. $\dot{M}<\dot{M}_{\text {max }}=\dot{M}_{\mathrm{Edd}}\left(r_{\mathrm{L}, \mathrm{ns}} / r_{\mathrm{ns}}\right)$, where $r_{\mathrm{ns}}$ is the radius of the neutron star (King \& Begelman 1999; Tauris et al. 2000). The dependence of $\dot{M}_{\max }$ on the mass of the donor is also plotted in Fig. 1. Since the mass-loss line in Fig. 1 holds as well for initial $M_{\mathrm{d}}<0.83 M_{\odot}$, within our assumptions "isotropic re-emission" from a neutron star is actually possible for donors with initial $M_{\mathrm{d}} \lesssim 0.44 M_{\odot}$. Unless there is an even more efficient way to stabilise the mass transfer than by "re-emission", this excludes ONe white dwarfs as donors as these have $M \gtrsim 1.1 M_{\odot}$ (Gil-Pons \& García-Berro 2001). Similarly also massive CO dwarfs are excluded as donors.

The overabundance (relative to solar) of Ne suggests, that the progenitors of the donors in the X-ray systems under consideration have experienced core helium burning including the ${ }^{14} \mathrm{~N}(\alpha, \gamma){ }^{18} \mathrm{~F}(\alpha, \gamma){ }^{22} \mathrm{Ne}$ reactions chain. Thus the donors cannot be low-mass helium white dwarfs.

This leaves as the last option the so called "hybrid" white dwarfs which have $\mathrm{CO}$ cores and thick He mantles. They are formed from components of close binaries with initial masses in the range from 2.5 to $5 M_{\odot}$ which experience RLOF prior to core He ignition, become He-burning stars and, after completion of core He burning, evolve directly into white dwarfs. Their mass is $\simeq(0.3-0.7) M_{\odot}$ (Iben \& Tutukov 1985; Han et al. 2000).

\footnotetext{
1 We neglect the effects of an X-ray irradiation induced stellar wind which may enhance mass loss by the donor (Tavani \& London 1993).
} 
In a calculation of the model of the population of compact stars in the Galaxy (Nelemans et al. 2001) the birthrate of such (wd, ns) systems with $M_{\mathrm{wd}} \leq 0.4 M_{\odot}$ is $2.7 \times 10^{-6} \mathrm{yr}^{-1}$. For comparison, the birthrate of progenitors of X-ray binaries with hydrogen-rich donors in the same model is $1.3 \times 10^{-5} \mathrm{yr}^{-1}$.

\subsection{Neon-enrichment}

Even after core helium exhaustion the abundance of $\mathrm{Ne}$ in the cores of hybrid white dwarfs is low, so to observe the strong Ne enrichment, the cores have to be crystallised and fractionated. In the models of white dwarfs these processes take several Gyr, depending on the mass of the dwarf, transparency of the outer layers, total amount of ${ }^{22} \mathrm{Ne}$ and uncertainties in phase diagrams (Hernanz et al. 1994; Montgomery et al. 1999). Figure 2 shows that the orbital periods of $(\mathrm{wd}, \mathrm{ns})$ pairs enter the observed range of $\sim 10 \mathrm{~min}$ in less than $10 \mathrm{Myr}$. (The meaning of the lines and asterisks in Fig. 2 is the same as in Fig. 1.) Hence, the enrichment of the white dwarf core by $\mathrm{Ne}$, must have happened before RLOF.

For an initial metallicity $Z=0.02$, after completion of He-burning, the mass abundance of ${ }^{22} \mathrm{Ne}$ in the core of the star cannot be larger than $X_{22 \mathrm{Ne}}=0.02$. During crystallisation of binary mixtures of $(\mathrm{C} / \mathrm{Ne})$ and $(\mathrm{O} / \mathrm{Ne})$, $\mathrm{Ne}$ settles in the center and forms a Ne-enriched nucleus which contains all ${ }^{22} \mathrm{Ne}$ formed in the dwarf and has the so called azeotropic abundance $X_{22 \mathrm{Ne}}^{\mathrm{a}}$ of $\mathrm{Ne}$ (Isern et al. 1991).

The mass of this Ne-rich nucleus is

$M_{\mathrm{Ne}-\mathrm{r}}=\left(X_{22 \mathrm{Ne}} / X_{22 \mathrm{Ne}}^{\mathrm{a}}\right) M_{\mathrm{wd}}$.

If, following Bravo et al. (1992), one assumes $X_{22 \mathrm{Ne}}^{\mathrm{a}}=$ 0.07 - an intermediate value of $X_{22 \mathrm{Ne}}^{\mathrm{a}}$ for $\mathrm{O} / \mathrm{Ne}$ and $\mathrm{C} / \mathrm{Ne}$ mixtures - and takes for $M_{\mathrm{wd}}$ the maximum mass of the convective core of a $0.378 M_{\odot}$ "hybrid" dwarf $-0.2 M_{\odot}$ (Iben \& Tutukov 1985), then the mass $M_{\mathrm{Ne}-\mathrm{r}}$ of the Neenriched core is $\simeq 0.06 M_{\odot}$.

If the white dwarf didn't have enough time to crystallise, the mass loss uncovers material of the convective core of the progenitor of the dwarf. Then the mass fraction of Ne may be down to the initial $\sim 0.001$.

\section{Comparison with the observed Ne-rich X-ray binaries}

\section{1. $4 U 0614+091,2 S 0918-549,4 U 1543-624$, and $4 \mathrm{U} 1850-0857$}

Figure 2 shows the evolution of a semidetached system which initially contained a "hybrid" white dwarf and a neutron star, in orbital period - mass loss rate coordinates. Taking the mass of the Ne-enriched core of $0.06 M_{\odot}$ estimated in Sect. 2.3 as typical, one obtains a rough lower limit of the orbital periods of binaries with the Ne-rich white dwarf donors of about $10 \mathrm{~min}$. The white dwarf
$\mathrm{CO} / \mathrm{He}$ donor+neutron star accretor

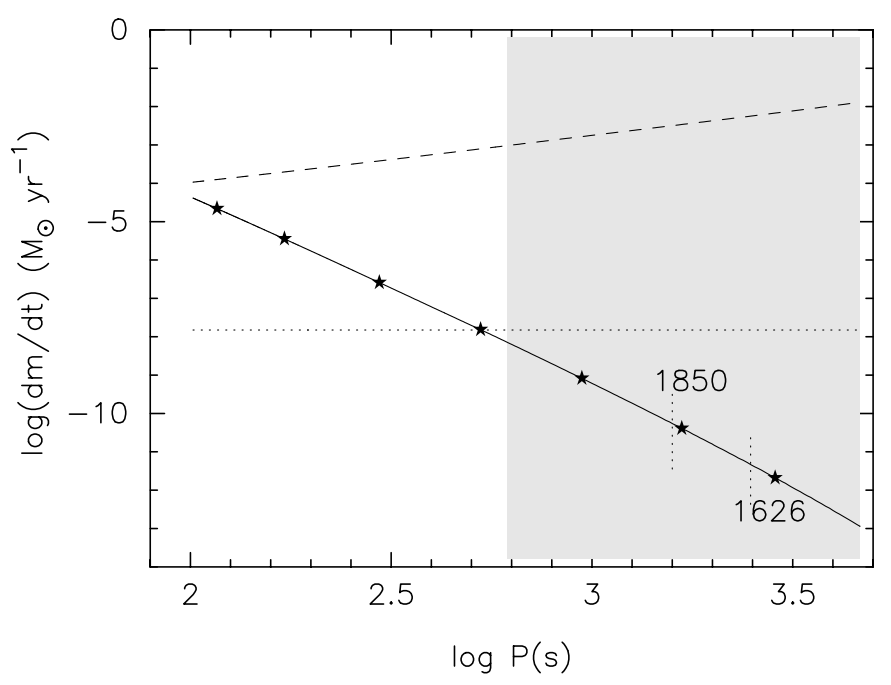

Fig. 2. Orbital period - mass loss rate relation for a system which initially contained a $0.38 M_{\odot}$ "hybrid" white dwarf donor and a $1.433 M_{\odot}$ neutron star accretor. The meaning of the lines and symbols is as in Fig. 1. The vertical dashed lines mark the orbital periods of $4 \mathrm{U} 1850-0857$ and $4 \mathrm{U}$ 1626-67. Within the period range corresponding to the shaded area the donors may transfer neon-enriched matter.

enters the range of periods (or masses) for which Neenrichment is possible in several Myr following the onset of RLOF (Fig. 2).

Our calculations of the mass loss rate also give the mass of the donor as a function of the orbital period. We thus infer the donor masses for $4 \mathrm{U}$ 1850-0857 and $4 \mathrm{U} 1626-67$ to be 0.027 and $0.01 M_{\odot}$, respectively (see more detailed discussion of the latter system in Sect. 3.2).

The abundance of Ne relative to solar found in the observed Ne-rich X-ray binaries ranges from $1.9 \pm 0.3$ to $2.87 \pm 0.16$. For oxygen an underabundance is reported: $0.37 \pm 0.06$ to $0.52 \pm 0.04$. Taken at face value, these relative abundances translate into a local $\mathrm{Ne} / \mathrm{O}$ ratio of 0.63-0.77. If true, these values are considerably higher than one would expect for the above used abundance of $\mathrm{Ne}$ in the nucleus of the dwarf $X_{22 \mathrm{Ne}}^{\mathrm{a}}=0.07$. But Isern et al. (1991) notice, that $X_{22 \mathrm{Ne}}^{\mathrm{a}}$ may be well underestimated by a factor $\sim 3$. If this is true, the $\mathrm{Ne} / \mathrm{O}$ ratio comes into better agreement with observed values. The mass of the Neenriched core then becomes slightly higher than $0.02 M_{\odot}$, still comparable with our estimates of the masses of the white dwarfs in $4 \mathrm{U}$ 1850-0857 and $4 \mathrm{U}$ 1626-67.

The problem of a low predicted $\mathrm{Ne} / \mathrm{O}$ ratio would also hold for initially more massive donors, if stable mass loss would be possible for them. In the core of a $1.1 M_{\odot} \mathrm{ONe}$ dwarf the Ne/O ratio is $\sim 0.4$ (Gil-Pons \& García-Berro 2001). For a "standard" $0.6 M_{\odot} \mathrm{CO}$ white dwarf with an initially equimolar distribution of $\mathrm{C} / \mathrm{O}$ and traces of $\mathrm{Ne}$, a final $\mathrm{Ne} / \mathrm{O}$ ratio $\sim 0.25$ is expected in the core (Segretain et al. 1994). 
All four Ne-enriched X-ray binaries contain, presumably, weakly magnetised neutron stars. This may be a result of the decay of the magnetic field by accretion (Taam \& van de Heuvel 1986).

\section{2. $4 U$ 1626-67: An accretion induced collapse?}

$4 \mathrm{U}$ 1626-67 differs from the binaries discussed above by the presence of a $7.7 \mathrm{~s}$ X-ray pulsar with a strong magnetic field estimated from cyclotron emission: $(3.2 \pm 0.1)(1+z) \times$ $10^{12} \mathrm{G}$, where $z$ is gravitational redshift (Orlandini et al. 1998). Applying a polytropic $M-R$ relation, Verbunt et al. (1990) estimated its donor mass as $\sim 0.02 M_{\odot}$. A similar estimate of $M_{\mathrm{d}}$ was obtained by Schulz et al. (2001) under the assumption that velocities of the lines in the $\mathrm{Ne} / \mathrm{O}$ complex and their Doppler shifts reflect Keplerian motions in the accretion disk. Thus, existing estimates of $M_{\mathrm{d}}$ are consistent with Ne-enrichment of the donors. A very low value of $M_{\mathrm{d}}$ in $4 \mathrm{U}$ 1626-67 is also favoured by the probability of detection considerations (Verbunt et al. 1990).

The high magnetic field strength suggests that the neutron star is young and accretion was negligible. The absence of enhanced abundances of O-group elements in the spectrum of $4 \mathrm{U}$ 1626-67 argues against the origin of the neutron star in a SN II event (Angelini et al. 1995). Taam \& van de Heuvel (1986) suggested that this X-ray pulsar was formed recently $-(2-10) \times 10^{7}$ yrs ago - by an accretion induced collapse (AIC) of a white dwarf. Verbunt et al. (1990) rejected the AIC model, based on the argument that the time between AIC and resumption of mass transfer is $\sim 10^{8} \mathrm{yr}$, longer than then assumed e-folding decay time $\left(\sim 10^{7} \mathrm{yr}\right)$ for the magnetic fields of neutron stars. Later, the analysis of magnetic fields of isolated radio pulsars have shown that the magnetic field decay scale is probably $\gtrsim 10^{8} \mathrm{yr}$ (Bhattacharya et al. 1992). This, along with the discovery of Ne-enrichment in the system, suggests that one should reconsider the formation of $4 \mathrm{U} 1626$ 67 via AIC, especially, since the details of pre-collapse evolution were never studied before.

In the model for the population of compact stars in the Galaxy (Nelemans et al. 2001) the birthrate of ONe white dwarfs with $M \geq 1.2 M_{\odot}$ accompanied by "hybrid" white dwarfs with $0.35 \leq M \leq 0.5 M_{\odot}$ which get into contact within $10 \mathrm{Gyr}$ is $1.25 \times 10^{-6} \mathrm{yr}^{-1}$. This rate is within the limits for occurrence of AIC's in the Galaxy set by nucleosynthesis considerations (Fryer et al. 1999).

The initial binary may contain e.g. a $0.38 M_{\odot}$ "hybrid" white dwarf (for which we know the internal structure from Iben \& Tutukov 1985) and a $1.2 M_{\odot}$ oxygen-neon one (Fig. 3). At the beginning of the RLOF $P_{\text {orb }}=2 \mathrm{~min}$. The outer $0.12 M_{\odot}$ of the donor consist of He. The maximum rate of stable He-burning at the surface of a massive white dwarf is about $10^{-5} M_{\odot} \mathrm{yr}^{-1}$ (Kato \& Hachisu 1999), lower than the mass loss rate by the donor in the initial stages of mass transfer. Mass exchange in this case may be also stabilised by isotropic re-emission of opti-
$4 \cup 1626-67$

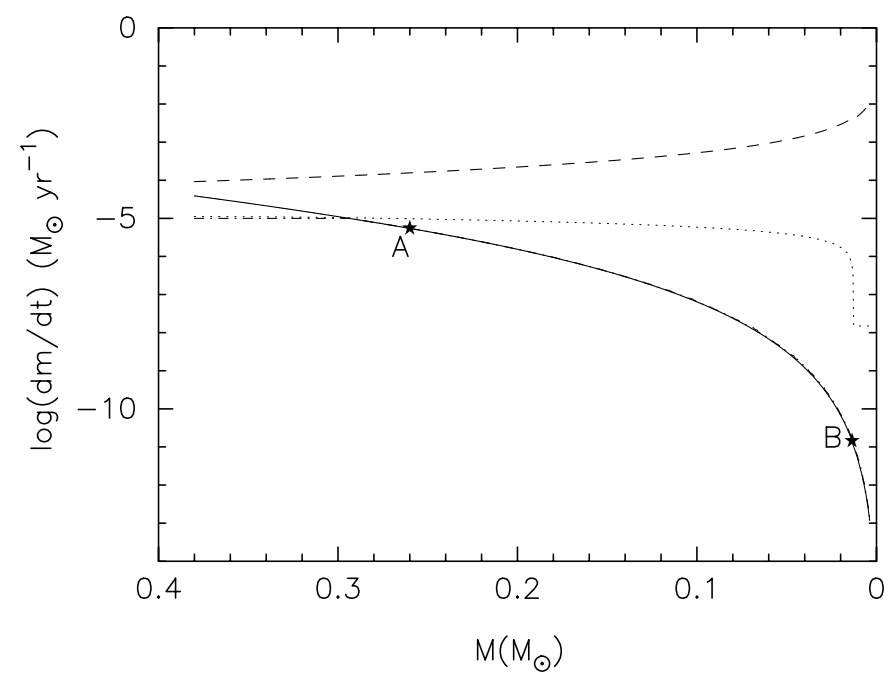

Fig. 3. Solid line - mass exchange rate in a system which initially consisted of a $0.38 M_{\odot}$ "hybrid" donor and a $1.2 M_{\odot} \mathrm{ONe}$ white dwarf accretor. The dashed line is the upper limit of the "isotropic re-emission" rate for an accreting white dwarf. The dot-dashed line - accretion rate. The dashed line $-\dot{M}_{\text {Edd }}$, first for a white dwarf, then for a neutron star. At point A the $\mathrm{He}$ mantle of the donor is exhausted, at point B the white dwarf collapses into a neutron star.

cally thick wind from accretor, generated by He-burning (Hachisu et al. 1996). Therefore stable helium burning is likely to set in on the surface of the $1.2 M_{\odot}$ accreting white dwarf.

Like Verbunt et al. (1990), we assume that the white dwarf collapses into a $1.26 M_{\odot}$ neutron star after the increase in mass to $1.44 M_{\odot}$. This happens after $240 \mathrm{Myrs}$ of accretion, when $P_{\text {orb }} \approx 2000 \mathrm{~s}$. The mass of the donor is at that moment $0.0132 M_{\odot}$. The $\mathrm{Ne} / \mathrm{O}$ ratio in the core for azeotropic abundance of $\mathrm{Ne}(0.1$ to 0.3 , see Sect. 2.3) doesn't contradict the measured $\mathrm{Ne} / \mathrm{O}$ ratio in $4 \mathrm{U}$ 1626-67 (Schulz et al. 2001): $0.22 \pm 0.15$.

The collapse interrupts mass transfer for $\sim 1.56 \times 10^{8} \mathrm{yr}$ due to the loss of binding energy of the dwarf (see for details Verbunt et al. 1990). This time span may not be sufficient for the decay of the magnetic field of the pulsar. When after resumption of the contact the period of the system has increased to $41.4 \mathrm{~min}$, the mass of the donor is $0.0101 M_{\odot}$.

Until this moment the neutron star in our model system has lived after the AIC for about 350 Myr. If magnetic fields do not decay on long time scale its field will not have decayed sufficiently. The accretion of $0.003 M_{\odot}$ onto a neutron star is most probably insufficient for destruction of its magnetic field (if the frozen field and incompressible fluid approximations (Cheng \& Zhang 1998) are assumed). Assuming solid body rotation and conservation of angular momentum, one gets $\simeq 1.3 \mathrm{~ms}$ for the initial spin period of the neutron star. The present spin period of the neutron 
star in $4 \mathrm{U} 1626-67$ is $7.7 \mathrm{~s}$, close to the "death-line" of radio pulsars for $B=3 \times 10^{12} \mathrm{G}$ (Bhattacharya 1996). Thus, it is conceivable that $4 \mathrm{U}$ 1626-67 harbours a neutron star which ceased to be a radio pulsar, but didn't experience recycling.

At the stage when our model system resembles $4 \mathrm{U} 1626-67$, the mass exchange rate is $\simeq 4 \times$ $10^{-12} M_{\odot} \mathrm{yr}^{-1}$. As already noticed before (Chakrabarty 1998; Schulz et al. 2001), all model $\dot{M}$ for $4 \mathrm{U}$ 1626-67 based on $M-R$ relations are considerably lower than the estimates of $\dot{M}$ based on the observed spin-up rate and simple angular momentum conservation considerations. It is plausible that the secular $\dot{M}$ is consistent with model expectations, while mass transfer rate $\dot{M}$ inferred from the observed spin-up of the pulsar (if correct) reflects the accretion rate from an unstable accretion disk (Schulz et al. 2001). The latter experiences thermal ionization instability if $\dot{M}_{\mathrm{a}} \lesssim 7.4 \times 10^{-10}$ and $\lesssim 2.5 \times 10^{-9} M_{\odot} \mathrm{yr}^{-1}$, for a pure $\mathrm{C}$ or $\mathrm{O}$ disk, respectively (Menou et al. 2002).

\section{Discussion}

White dwarfs may be considered as a ternary $\mathrm{C} / \mathrm{O} / \mathrm{Ne}$ ionic mixture. For such mixtures Ogata et al. (1993) predict the formation of an almost pure Ne core upon solidification for any $\mathrm{C}$ :O ratio. If this core ultimately contains all ${ }^{22} \mathrm{Ne}$ generated in the white dwarf its mass is only $\sim 0.004 M_{\odot}$, much less than given by Eq. (1) and this would require much larger $P_{\text {orb }}$ for X-ray binaries showing Ne enrichment.

On the other hand, according to Segretain (1996), if a $0.6 M_{\odot}$ white dwarf is considered as a ternary mixture with $X_{\mathrm{C}}=X_{\mathrm{O}}=0.495, X_{22 \mathrm{Ne}}=0.01$, almost all Ne concentrates in a thin layer around $M_{\mathrm{r}} / M_{\mathrm{wd}} \approx 0.7$. Then spilling of Ne-enriched matter over the neutron star would require extremely fine tuning of the model.

Another intriguing problem concerns X-ray bursts. They were reported for $4 \mathrm{U} 0614+091,2 \mathrm{~S} 0918-549$, and $4 \mathrm{U} 1850-0857$. None of the bursts have shown atypical behaviour (Juett et al. 2001). Carbon flashes at $\dot{M}<$ $0.1 \dot{M}_{\text {Edd }}$ were never studied. Extrapolation of the calculations of Cumming \& Bildsten (2001) over 2 orders of magnitude may be not very relevant. However, it shows that for $X_{\mathrm{C}} \sim 0.2$, characteristic for "hybrid" dwarfs and $\dot{M} \sim 10^{-11} M_{\odot} \mathrm{yr}^{-1}$, accreted carbon possibly burns stably. Thus, the origin of the bursts in systems with any low-mass C-rich donors deserves further attention.

\section{Conclusion}

We have shown that within the framework of the white dwarfs cooling model developed by Isern et al. (1991), Bravo et al. (1992), Segretain et al. (1994), Hernanz et al. (1994) it is possible to explain the formation of the Neenriched donors in X-ray binaries if their progenitors were low-mass $\mathrm{CO}$ white dwarfs with thick He mantles. A necessary condition is that the white dwarfs had enough time to be substantially chemically fractionated. This means that the mass transfer had to start several Gyr after the formation of the white dwarfs. We estimate that the masses of Ne-rich donors in these systems are $\lesssim 0.06 M_{\odot}$ and their orbital periods have to be $\gtrsim 10 \mathrm{~min}$. The expected Ne/O ratios are $\sim(0.1-0.4)$.

Finally we conclude that the X-ray pulsar 4U 1626-67 could follow an evolutionary path similar to the other Nerich systems, with the only difference that it used to have a massive ONe white dwarf component that collapsed into a neutron star relatively recently. We estimate the current mass of the donor in this system as $0.01 M_{\odot}$.

Acknowledgements. We acknowledge fruitful discussions with A. Kudrjashoff, O. Pols, G.-J. Savonije, E. García-Berro, J. Isern, R. Ramachandran. Our thanks a due to Z. Han for communicating his unpublished results. LRY acknowledges the warm hospitality and support of the Astronomical Institute "Anton Pannekoek". This work was supported by a NOVA grant, NWO Spinoza grant 08-0 to E. P. J. van den Heuvel, and "Astronomy" Program.

\section{References}

Angelini, L., White, N. E., Nagase, F., et al. 1995, ApJ, 449, L41

Bhattacharya, D. 1996, in Compact Stars in Binaries, IAU Symp., 165, 243

Bhattacharya, D., Wijers, R. A. M. J., Hartman, J. W., \& Verbunt, F. 1992, A\&A, 254, 198

Bravo, E., Isern, J., Canal, R., \& Labay, J. 1992, A\&A, 257, 534

Chakrabarty, D. 1998, ApJ, 492, 342

Cheng, K. S., \& Zhang, C. M. 1998, A\&A, 337, 441

Cumming, A., \& Bildsten, L. 2001, ApJ, 559, L127

Fryer, C., Benz, W., Herant, M., \& Colgate, S. A. 1999, ApJ, 516,892

Gil-Pons, P., \& García-Berro, E. 2001, A\&A, 375, 87

Hachisu, I., Kato, M., \& Nomoto, K. 1996, ApJ, 470, L97

Han, Z., Tout, C. A., \& Eggleton, P. P. 2000, MNRAS, 319, 215

Hernanz, M., García-Berro, E., Isern, J., et al. 1994, ApJ, 434, 652

Iben, I. Jr., \& Tutukov, A. V. 1985, ApJS, 58, 661

Iben, I. Jr., Tutukov, A. V., \& Yungelson, L. R. 1995, ApJS, 100, 233

Isern, J., Hernanz, M., Mochkovitch, R., \& García-Berro, E. 1991, A\&A, 241, L29

Juett, A. M., Psaltis, D., \& Chakrabarty, D. 2001, ApJ, 560, L59

Kato, M., \& Hachisu, I. 1999, ApJ, 513, L41

King, A. R., \& Begelman, M. C. 1999, ApJ, 519, L169

Menou, K., Perna, R., \& Hernquist, L. 2002, ApJ, 564, L81

Montgomery, M. H., Klumpe, E. W., Winget, D. E., \& Wood, M. A. 1999, ApJ, 525, 482

Nelemans, G., Yungelson, L. R., \& Portegies Zwart, S. F. 2001, A\&A, 375, 890

Ogata, S., Iyetomi, H., Ichimaru, S., \& van Horn, H. M. 1993, Phys. Rev. E, 48, 1344

Orlandini, M., Fiume, D. D., Frontera, F., et al. 1998, ApJ, 500, L163

Paczyński, B. 1967, Acta Astron., 17, 287 
Panei, J. A., Althaus, L. G., \& Benvenuto, O. G. 2000, A\&A, 353,970

Rappaport, S., Ma, C. P., Joss, P. C., \& Nelson, L. A. 1987, ApJ, 322, 842

Rasio, F. A., Pfahl, E. D., \& Rappaport, S. 2000, ApJ, 532, L47

Schulz, N. S., Chakrabarty, D., Marshall, H. L., et al. 2001, ApJ, 563, 941

Segretain, L. 1996, A\&A, 310, 485

Segretain, L., Chabrier, G., Hernanz, M., et al. 1994, ApJ, 434, 641

Soberman, G. E., Phinney, E. S., \& van den Heuvel, E. P. J. 1997, A\&A, 327, 620

Taam, R. E., \& van de Heuvel, E. P. J. 1986, ApJ, 305, 235
Tauris, T. M., \& Savonije, G. J. 1999, A\&A, 350, 928

Tauris, T. M., van den Heuvel, E. P. J., \& Savonije, G. J. 2000, ApJ, 530, L93

Tavani, M., \& London, R. 1993, ApJ, 410, 281

Tutukov, A. V., \& Yungelson, L. R. 1979, Acta Astron., 29, 665

Tutukov, A. V., \& Yungelson, L. R. 1993, ARep, 37, 411

Verbunt, F., Wijers, R. A. M. J., \& Burm, H. M. G. 1990, A\&A, 234, 195

Vila, S. C. 1971, ApJ, 168, 217

Yungelson, L., Livio, M., Truran, J. W., Tutukov, A., \& Fedorova, A. 1996, ApJ, 466, 890

Zapolsky, H. S., \& Salpeter, E. E. 1969, ApJ, 158, 809 